\title{
Requeijão com teor reduzido de cloreto de sódio: caracterização físico-química e sensorial entre adolescentes
}

\author{
Cottage cheese content reduced sodium chloride: physico-chemical and sensory \\ characterization among teenagers
}

\author{
Graziela Nunes ${ }^{1}$ \\ Flávia Teixeira² \\ Mirelly Marques Romeiro ${ }^{3}$ \\ Camila Jordão Candido ${ }^{4}$ \\ Priscila Aiko Hiane ${ }^{5}$ \\ Anderson Fernandes da Silva ${ }^{6}$ \\ Danieli Fernanda Zampieri ${ }^{7}$ \\ Elisvânia Freitas dos Santos ${ }^{8}$ \\ Daiana Novello $\left.{ }^{9}{ }^{*}\right)$
}

\section{Resumo}

O objetivo do trabalho foi verificar a aceitabilidade sensorial e a composição físicoquímica de requeijões elaborados com diferentes teores de cloreto de sódio, cloreto de potássio e glutamato monossódico, entre adolescentes. Na Etapa 1 foram formuladas

1 Me.; Desenvolvimento Comunitário; Universidade Estadual do Centro-Oeste, UNICENTRO, Brasil; Nutricionista; Guarapuava, PR; Endereço: Universidade Estadual do Centro-Oeste, Curso de Nutrição, R. Simeão Varela de Sá, 03 Vila Carli, CEP: 85040-080 - Guarapuava, PR - Brasil; E-mail: grazielaznunes@hotmail.com

2 Me.; Desenvolvimento Comunitário; Universidade Estadual do Centro-Oeste, UNICENTRO, Brasil; Nutricionista; Guarapuava, PR; Endereço: Universidade Estadual do Centro-Oeste, Curso de Nutrição, R. Simeão Varela de Sá, 03 Vila Carli, CEP: 85040-080 - Guarapuava, PR - Brasil; E-mail: teixeiraflavia19@gmail.com

3 Dra.; Saúde e Desenvolvimento na Região Centro-Oeste; Universidade Federal de Mato Grosso do Sul, UFMS, Brasil; Nutricionista, Campo Grande, MS; Endereço: Universidade Federal de Mato Grosso do Sul, Centro de Ciências Biológicas e da Saúde da UFMS, Departamento de Tecnologia de Alimentos e Saúde Pública. Cidade Universitária, CEP:79070-900 - Campo Grande, MS - Brasil; E-mail: mirellymarques@hotmail.com

4 Me.; Saúde e Desenvolvimento na Região Centro-Oeste; Universidade Federal de Mato Grosso do Sul, UFMS, Brasil; Técnica em Alimentos e Laticínios da UFMS atuando na área de ciência e tecnologia de alimentos, química dos alimentos e nutrição experimental. Professora colaboradora de bromatologia e bioquímica de alimentos; Endereço: Universidade Federal de Mato Grosso do Sul, Conselho de Pesquisa e Pós-Graduação da UFMS. Cidade Universitária, CEP:79070900 - Campo Grande, MS - Brasil; E-mail: cahjordao@gmail.com

5 Dra.; Ciências da Saúde; Universidade de Brasília, UnB, Brasil; Professora Titular da Universidade Federal de Mato Grosso do Sul (UFMS); Endereço: Universidade Federal de Mato Grosso do Sul, Centro de Ciências Biológicas e da Saúde da UFMS, Departamento de Tecnologia de Alimentos e Saúde Pública. Cidade Universitária. CEP: 79070900 Campo Grande, MS - Brasil; E-mail: priscila.hiane@ufms.br

6 Dr.; Saúde e Desenvolvimento na Região Centro-Oeste; Universidade Federal de Mato Grosso do Sul, UFMS, Brasil; Professor na Universidade Estadual de Mato Grosso do Sul (UEMS) - Unidade Coxim; Endereco: Universidade Federal de Mato Grosso do Sul. Rua Filinto Muller s/n, Cidade Universitária - FAMED - Laboratório de Metabolismo Mineral e Biomateriais, Ipiranga. CEP: 79070900 - Campo Grande, MS - Brasil; E-mail: fernandes.centrooeste@hotmail.com

7 Me.; Ciências de Alimentos; Universidade Estadual de Londrina, UEL, Brasil; Técnica em alimentos e laticínios da UFMS; Endereço: Universidade Federal de Mato Grosso do Sul, Centro de Ciências Biológicas e da Saúde da UFMS, Departamento de Tecnologia de Alimentos e Saúde Pública. Cidade Universitária, CEP: 79070-900 - Campo Grande, MS - Brasil; E-mail: dani_zampieri@hotmail.com

8 Dra.; Ciências da Cirurgia; Universidade Estadual de Campinas, UNICAMP, Brasil; Professora Adjunta III do Curso de Nutrição da Universidade Federal de Mato Grosso do Sul (UFMS) e Professora Colaboradora do Programa de Pós Graduaçã̃o em Saúde e Desenvolvimento na Região Centro-Oeste da Faculdade de Medicina (FAMED) e do Programa de Pós Graduação em Biotecnologia da UFMS; Endereço: Universidade Federal de Mato Grosso do Sul, Centro de Ciências Biológicas e da Saúde. CCBS/DTA/Nutrição, Cidade Universitária. CEP: 79070900 - Campo Grande, MS Brasil; E-mail: elisvania@gmail.com

9 Dra.; Tecnologia de Alimentos; Nutricionista; Universidade Estadual de Campinas,UNICAMP,Brasil; Professora Adjunta D do Curso de Nutrição da Universidade Estadual do Centro-Oeste (UNICENTRO) e Professora do Programa de Pós Graduação Interdisciplinar em Desenvolvimento Comunitário (PPGDC) da UNICENTRO; Endereço: Universidade Estadual do Centro-Oeste, Curso de Nutrição, R. Simeão Varela de Sá, 03 - Vila Carli, CEP: 85040-080 - Guarapuava, PR - Brasil; E-mail: nutridai@gmail.com. $\left(^{*}\right)$ Autor para correspondência.

$\begin{array}{llllll}\text { Ambiência } & \text { Guarapuava (PR) } & \text { v.l4 n.3 } & \text { p.662 - 677 } & \text { Set/Dez } 2018 & \text { ISSN } 1808-025 \text { I }\end{array}$


cinco amostras de requeijão adicionadas de cloreto de sódio e cloreto de potássio, em conjunto ou individualmente, nas porcentagens de 0 a 0,40\%. Na Etapa 2 utilizaram-se as mesmas porcentagens de adição da Etapa 1, porém com acréscimo de 0,30\% de glutamato monossódico. Na Etapa 1, não houve diferença significativa entre as formulações para os atributos aparência, aroma e cor. Porém, a amostra contendo 0,40\% de cloreto de potássio apresentou menor aceitação para os atributos sabor, sabor residual, intenção de compra e aceitação global, sendo que a adição de até 0,30\% de cloreto de potássio apresentou aceitabilidade similar a padrão em todos os atributos avaliados. $\mathrm{Na}$ Etapa 2, com a adição de glutamato monossódico, houve um aumento geral nas notas dos produtos. Foi possível restringir o teor de cloreto de sódio em requeijão, principalmente através da utilização conjunta do cloreto de sódio $(0,10 \%)$ e potássio $(0,30 \%)$, sendo ainda plausível a utilização de glutamato monossódico para melhoramento da aceitação sensorial.

Palavras-chave. aceitabilidade; redução de sódio; sais.

\section{Abstract}

The aim of work was to evaluate the sensory acceptability and physico-chemical composition of cottage cheese with different amounts of sodium chloride, potassium chloride and monosodium glutamate, among adolescents. In Step 1 were formulated five samples of cottage cheese added sodium chloride and potassium chloride, individually or together, in percentages of 0 to $0.40 \%$. In Step 2 we used the same percentages added in Step 1, but with addition of $0.30 \%$ of monosodium glutamate. In Step 1 there was no significant difference between formulations for attributes appearance, aroma and color. However, the sample containing $0.40 \%$ potassium chloride was less well accepted for the attributes taste, aftertaste, purchase intent and global acceptance, with the addition of up to $0.30 \%$ potassium chloride had similar acceptability standard in all evaluated attributes. In Step 2. With the addition of monosodium glutamate, there was a general increase in the notes of the products. It was possible to limit the sodium chloride content of the cottage cheese, particularly through the combined use of sodium chloride $(0.10 \%)$ and potassium $(0.30 \%)$, being a plausible the use of monosodium glutamate for improving sensory acceptance.

Keywords. acceptability; reduced sodium; salts.

\section{Introdução}

A hipertensão arterial sistêmica (HAS) se destaca como um dos principais fatores de risco para doenças cardiovasculares. Estas patologias predominam como causadoras de mortalidade no Brasil e no mundo, pois afetam, aproximadamente, um terço da população mundial(OMS, 2015). Dentre os grupos mais acometidos pela HAS, na última década, estão crianças e adolescentes, que apresentam maior risco potencial à saúde (CAMPANA et al., 2009). Conceitualmente, a HAS se caracteriza, principalmente, pelo aumento da pressão arterial sanguínea, quando os níveis são iguais ou superiores a 140 / $90 \mathrm{mmHg}$ (TRAD et al., 2010).

Devido a sua praticidade e acessibilidade, admite-se que o alto consumo de alimentos processados, com elevados teores de cloreto de sódio $(\mathrm{NaCl})$, seja um dos principais agentes 
causadores da HAS (RIBEIRO et al., 2013). A ingestão de sódio recomendada para adultos corresponde a $2.000 \mathrm{mg} /$ dia ou $5 \mathrm{~g}$ de $\mathrm{NaCl}$ (WHO, 2011). Porém, esse valor reduzido em caso de adolescentes $(1,5 \mathrm{~g}$ de $\mathrm{NaCl} / \mathrm{dia})$, visto que são parte de uma população mais vulnerável (COZZOLINO, 2012). Nesse contexto, são necessárias ações globais que visem à prevenção dessa patologia, principalmente no que se refere à mudança de hábitos alimentares e consumo de alimentos mais saudáveis. Entre as principais estratégias para atingir este objetivo destacamse a redução voluntária de sódio da dieta e a execução de campanhas nacionais e internacionais (WHO, 2010).

O governo brasileiro, desde 2010, em parceria com o Ministério da Saúde, Agência Nacional de Vigilância Sanitária (ANVISA) e instituições e organizações privadas, vem realizando seminários com o objetivo de estabelecer ações e medidas para reduzir o consumo de sódio. Dentre elas estão o aumento da oferta de alimentos saudáveis, atividades de orientação para a população, profissionais de saúde e manipuladores de alimentos sobre o uso correto da rotulagem nutricional (NILSON et al.,2012). Alguns temas apresentam maior enfoque, como o consumo de alimentos in natura e reformulação de alimentos processados, para que contenham menor teor de gordura, açúcar e sódio (MONTEIRO et al., 2011).

Atualmente, são conhecidos mais de 500 tipos de queijos e novas variedades têm surgido no mercado (EL-BAKRY e SHEEHAN, 2014). O requeijão ocupa posição de destaque nas vendas e consumo de lácteos, contudo, é uma fonte significativa de gordura e sódio, contendo cerca de $620 \mathrm{mg}$ de sódio/ $100 \mathrm{~g}$ de produto (AGARWAL et al., 2011). Assim, a redução de $\mathrm{NaCl} \mathrm{em}$ produtos como o requeijão apresenta-se como um grande desafio para a indústria alimentícia. Esse fato se deve às funções específicas do sódio na produção e conservação desse tipo de produto, como a textura e prazo de validade (PURDY e ARMSTRONG,2007). Contudo, alguns estudos mostram que é possível reduzir o teor de $\mathrm{NaCl}$ em uma porcentagem de no mínimo $25 \%$, sem prejudicar as características tecnológicas e sensoriais do requeijão (VAN DENDER et al.,2012). Deste modo, novas pesquisas que visem avaliar produtos alimentícios, como os queijos com baixo teor de sódio, representam uma contribuição para a indústria, visto que também colaboram para a saúde e maior valor econômico e tecnológico (DRAKE et al., 2011). Contudo, são necessárias avaliações constantes de suas características, minimizando o impacto de possíveis alterações nos produtos.

O cloreto de potássio $(\mathrm{KCl})$ é um dos sais substitutos do $\mathrm{NaCl}$ mais utilizados, por ser considerado seguro e apresentar propriedades antimicrobiológicas semelhantes as do $\mathrm{NaCl}$ (BIDLAS e LAMBERT, 2008). Além disso, a ingestão adequada de sódio e potássio colabora para o equilíbrio hídrico e regulação da pressão osmótica do organismo, contribuindo como um fator protetor ao aumento da pressão sanguínea (COZZOLINO,2012). Porém, reduções parciais ou totais de $\mathrm{NaCl}$ e adição de $\mathrm{KCl}$ em alimentos e produtos, podem trazer algumas desvantagens. Por exemplo, a redução na sensação do sabor salgado, diminuição da estabilidade microbiológica ou, ainda, anomalias na cor e textura e uma possível introdução de sabores metálicos, amargos e/ ou adstringentes (ALINO et al., 2009). Assim, recomenda-se a combinação destes minerais nas formulações, reduzindo-se os efeitos negativos, os quais também podem ser amenizados com o uso de intensificadores de sabor como o glutamato monossódico (GM). Este realçador é utilizado nos alimentos com frequência, a fim de salientar os sabores salgados, transmitidos por meio do ácido glutâmico, que ocorre naturalmente em alimentos protéicos (RANGAN e BARCELOUX, 2009). 
As características sensoriais de um produto definem sua qualidade sensorial e, consequentemente, a sua aceitação ou rejeição pelos consumidores. A análise sensorial, através dos sentidos humanos, utiliza métodos específicos que avaliam as sensações obtidas, permitindo concluir sobre a qualidade e aceitabilidade do alimento (DUTCOSKY, 2011). Já, a análise físico-química avalia produtos objetivando a identificação de fraudes ou adulterações, alterações físico-químicas, quantificação de aditivos alimentares e adequação ao Padrão de Identidade e Qualidade (PIQ) e rotulagem nutricional (ANDRADE, 2012), visando garantir as características dos alimentos (GOMES et al., 2011). Assim, para que novos produtos alimentícios, alterados em sua composição e/ ou quantidade de ingredientes, sejam comercializados no mercado, é fundamental a aplicação desses testes científicos. Diante do exposto, o objetivo deste trabalho foi verificar a aceitabilidade sensorial de requeijões elaborados com diferentes teores de $\mathrm{NaCl}$, $\mathrm{KCl}$ e GM, entre adolescentes, e avaliar a composição físico-química do produto.

\section{Materiais e métodos}

\section{Matéria-prima}

Os ingredientes básicos utilizados para as formulações foram adquiridos em supermercados do município de Guarapuava, PR. Os sais foram doados por empresas parceiras e apresentavam a seguinte classificação: $\mathrm{NaCl}$ (para análise, P.A.), peso molecular 58,44 e; $\mathrm{KCl}$ (P.A.), peso molecular 74,55. O GM utilizado foi um produto comercial, contendo $12.300 \mathrm{mg} \mathrm{Na} / 100 \mathrm{~g}$.

\section{Preparo das formulações}

O preparo do requeijão foi realizado no Laboratório de Técnica e Dietética do curso de Nutrição da UNICENTRO. Os seguintes ingredientes foram utilizados para a formulação base: leite integral (80,52\%); vinagre branco (1,61\%); creme de leite $(16,10 \%)$; margarina sem sal $(1,13 \%)$ e amido de milho (0,24\%), todos adquiridos em um supermercado local da cidade de Guarapuava, PR. Na Etapa 1, foram elaboradas 5 formulações de requeijão com diferentes teores de $\mathrm{NaCl}$ e $\mathrm{KCl}$ sendo: $\mathrm{F} 1$ - (0,40\% de $\mathrm{NaCl}-100 \%), \mathrm{F} 2$ (0,30\% de $\mathrm{NaCl}-75 \%$ e $0,10 \%$ de $\mathrm{KCl}$ - 25\%), F3 (0,20\% de $\mathrm{NaCl}-50 \%$ e 0,20\% de $\mathrm{KCl}-50 \%)$, F4 (0,10\% de $\mathrm{NaCl}-25 \%$ e $0,30 \%$ de $\mathrm{KCl}-75 \%)$ e $\mathrm{F} 5$ (0,40\% de $\mathrm{KCl}$ - 100\%). Na Etapa 2, utilizaram-se basicamente as mesmas porcentagens de adição de sais da Etapa 1, porém foram acrescidas, a cada amostra, o realçador de sabor GM na porcentagem de 0,30\%, totalizando-se 5 formulações, sendo elas: F1 (0,40\% de $\mathrm{NaCl}+0,30 \%$ de GM), F2 (0,30\% de $\mathrm{NaCl}$ e $0,10 \%$ de $\mathrm{KCl}+0,30 \%$ de GM), F3 (0,20\% de $\mathrm{NaCl}$ e $0,20 \%$ de $\mathrm{KCl}+0,30 \%$ de $\mathrm{GM}), \mathrm{F} 4(0,10 \%$ de $\mathrm{NaCl}$ e $0,30 \%$ de $\mathrm{KCl}$ + 0,30\% de GM) e F5 (0,40\% de $\mathrm{KCl}$ + 0,30\% de GM). Os níveis de adição estabelecidas nas Etapas 1 e 2 foram definidos através de testes sensoriais preliminares realizados com o produto. Os ingredientes foram pesados em balança portátil (Filizola ${ }^{\circledR}$, Brasil), com capacidade de $5 \mathrm{~kg}$.

O leite foi fervido em chama média $\left(180^{\circ} \mathrm{C}\right)$, por 5 minutos e, em seguida, reservada uma quantidade de 5,38\%. Na porção restante $(94,62 \%)$, adicionou-se o vinagre, sendo misturado vagarosamente por 5 minutos até coalhar. A mistura foi coada com o auxílio de uma peneira caseira até escoamento total dos líquidos, assim obteve-se a ricota. Em um liquidificador (Mondial ${ }^{\oplus}$, Brasil) misturou-se o creme de leite, a margarina, ricota, amido de milho, o leite e o sal, até se obter consistência homogênea. A mistura foi levada ao fogo em chama moderada $\left(160^{\circ} \mathrm{C}\right)$, por aproximadamente 2 minutos. Posteriormente, o requeijão foi acondicionado em recipiente de vidro e armazenado em geladeira $\left(8^{\circ} \mathrm{C}\right)\left(\mathrm{Consul}^{\circledR}, \mathrm{Brasil}\right)$, por cerca de uma hora, obtendo maior consistência. 


\section{Análise sensorial}

Participaram da análise sensorial 240 adolescentes (60 indivíduos em cada teste sensorial), matriculados em uma escola pública do município de Guarapuava, PR. Os provadores foram de ambos os gêneros, não treinados, com idade entre 14 e 17 anos.

A avaliação sensorial foi realizada em uma sala da escola, em cabines individuais, sob luz natural. Foram avaliados os atributos de aparência, aroma, sabor, sabor residual e cor. As amostras foram analisadas por meio de uma escala hedônica estruturada de 9 pontos, variando de "desgostei muitíssimo" (nota 1) a "gostei muitíssimo" (nota 9). Foram aplicadas também questões de aceitação global com auxílio de escala hedônica estruturada de 9 pontos e intenção de compra, utilizando-se uma escala estruturada de 5 pontos (1: "certamente não compraria", 5: "certamente compraria") (DUTCOSKY, 2011).

Cada julgador recebeu uma porção das formulações (aproximadamente $5 \mathrm{~g}$ ), em copos plásticos brancos codificados com números de três dígitos, de forma balanceada e casualizada, acompanhada de água para realização do branco. As formulações foram oferecidas aos julgadores de forma monádica sequencial.

Um teste de ordenação foi aplicado para comparar as diferenças das amostras em atributo específico de sabor salgado, já que o requeijão foi reduzido em sódio. Nesse teste, os provadores classificaram as amostras em ordem crescente de sabor salgado, sendo da menos salgada para a mais salgada (DUTCOSKY, 2011).

O cálculo do índice de aceitabilidade (IA) das formulações foi realizado segundo a fórmula: $I A(\%)=A \times 100 / B$ ( $A$ = nota média obtida para o produto; $B=$ nota máxima dada ao produto) (DUTCOSKY, 2011).

\section{Composição físico-química}

As análises físico-químicas foram realizadas no Laboratório de Físico-Química da Unidade de Tecnologia de Alimentos e Saúde Pública (UTASP) da UFMS. As análises de umidade e cinzas (AOAC, 2011) foram realizadas em triplicata, em todas as formulações padrão dos requeijões, devido à variação no teor de sais dos produtos. As demais determinações foram realizadas, em triplicata, na formulação padrão dos requeijões: Lipidios totais: Utilizou-se o método de extração a frio (BLIGH e DYER, 1959); Proteinas: Foram avaliadas através do teor de nitrogênio total da amostra, pelo método Kjeldahl, determinado ao nível semimicro (AOAC, 2011). Utilizou-se o fator de conversão de nitrogênio para proteína de 6,25; Fibra alimentar: Foi realizado o cálculo teórico através do programa Avanutri versão 3.0; Carboidratos: A determinação de carboidratos dos produtos foi realizada através de cálculo teórico (por diferença) nos resultados das triplicatas, conforme a fórmula: \% Carboidratos $=100-(\%$ umidade $+\%$ proteina $+\%$ lipidios $+\%$ cinzas $+\%$ fibra alimentar); Valor calórico: $\mathrm{O}$ total de calorias (kcal) foi calculado utilizando-se os seguintes valores: lipídios $(8,93 \mathrm{kcal} / \mathrm{g})$, proteína $(4,27 \mathrm{kcal} / \mathrm{g})$ e carboidratos $(3,82 \mathrm{kcal} / \mathrm{g})$ (MERRILL e WATT, 1973).

Os minerais ferro, magnésio, cálcio e fósforo foram determinados no Laboratório de Metabolismo Mineral e Biomateriais da UFMS. As amostras foram digeridas com $\mathrm{HNO}_{3} \mathrm{e}_{2} \mathrm{O}_{2}$ em frascos fechados de Hostaflon, pressurizados e com aquecimento em digestor (Thermo Fisher Scientific, Cambridge, Inglaterra). Após a digestão, a solução resultante foi transferida para um frasco tipo falcon, sendo o volume completado com água deionizada. Para a determinação de minerais foi utilizada espectrometria de emissão ótica com plasma indutivamente acoplado-ICP OES (Thermo Fisher Scientific, Cambridge, Inglaterra), modelo iCAP 6300 Duo (POUSSEL e MERMET, 1995). 
Determinação do valor diário de referência (VD)

O VD foi calculado em relação a $30 \mathrm{~g}$ da amostra, com base nos valores médios preconizados para adolescente (14 a 17 anos) (DRI, 2005), resultando em: 2.214,00 kcal/ dia, 299,00 g/ dia de carboidratos, 77,33 g/ dia de proteínas, 81,00 g/ dia de lipídios, 14,43 g/ dia de fibra alimentar. O VD para os micronutrientes foi calculado considerando 2.000,00 mg/ dia de sódio (WHO, 2011) e 2.455,00 mg/ dia de potássio (DRI, 2005).

Análise estatística

Os dados foram avaliados através da análise de variância (ANOVA), utilizando-se o teste de Tukey e teste de student para comparação de médias. No teste de ordenação foram empregados também o Teste de Friedman e a Tabela de Christensen, que indicaram a diferença mínima significativa (DMS) entre as amostras testadas e o número de julgamentos obtidos (DUTCOSKY, 2011). Todos os testes foram analisados em nível de 5\% de significância, com auxílio do software Statgraphics plus ${ }^{\oplus}$, versão 5.1.

Questões éticas

Este trabalho foi aprovado pelo Comitê de Ética em Pesquisa da UNICENTRO, parecer no 345.569/2013. Como critérios de exclusão foram considerados os seguintes fatores: indivíduos com idade superior a 17 anos ou menores de 14 anos, não ser aluno da escola em questão ou não entregar o Termo de Consentimento Livre e Esclarecido (TCLE) assinado pelo responsável legal.

\section{Resultados e discussão}

Análise sensorial

$\mathrm{Na}$ Tabela 1 encontram-se as médias dos testes sensoriais afetivo, de intenção de compra e de ordenação e IA dos requeijões formulados na Etapa 1 e 2.

Tabela 1. Índices de aceitabilidade (IA) e médias dos testes sensorial afetivo, de intenção de compra e de ordenação (sabor salgado) realizados para as formulações de requeijão adicionadas de cloreto de sódio ( $\mathrm{NaCl}$ ), cloreto de potássio ( $\mathrm{KCl}$ ) e glutamato monossódico (GM)

\begin{tabular}{cccccc}
\hline $\begin{array}{c}\text { Amostras/ } \\
\text { Atributos }\end{array}$ & $\begin{array}{c}\text { F1 } \\
\text { Média } \pm \text { EPM }\end{array}$ & $\begin{array}{c}\text { F2 } \\
\text { Média } \pm \text { EPM }\end{array}$ & $\begin{array}{c}\text { F3 } \\
\text { Média } \pm \text { EPM }\end{array}$ & $\begin{array}{c}\text { F4 } \\
\text { Média } \pm E P M\end{array}$ & $\begin{array}{c}\text { F5 } \\
\text { Média } \pm E P M\end{array}$ \\
\hline Etapa 1 & & & & & \\
Aparência & $7,02 \pm 0,15^{\mathrm{a}}$ & $7,02 \pm 0,17^{\mathrm{a}}$ & $7,12 \pm 0,18^{\mathrm{a}}$ & $7,43 \pm 0,15^{\mathrm{a}}$ & $7,02 \pm 0,23^{\mathrm{a}}$ \\
IA (\%) & $78,00 \%$ & $78,00 \%$ & $79,11 \%$ & $82,55 \%$ & $78,00 \%$ \\
Aroma & $6,75 \pm 0,18^{\mathrm{a}}$ & $6,53 \pm 0,23^{\mathrm{a}}$ & $6,55 \pm 0,28^{\mathrm{a}}$ & $6,70 \pm 0,24^{\mathrm{a}}$ & $6,36 \pm 0,26^{\mathrm{a}}$ \\
IA (\%) & $75,00 \%$ & $72,55 \%$ & 72,78 & 74,44 & 70,66 \\
Sabor & $6,90 \pm 0,18^{\mathrm{a}}$ & $6,60 \pm 0,26^{\mathrm{a}}$ & $6,62 \pm 0,30^{\mathrm{a}}$ & $6,00 \pm 0,32^{\mathrm{ab}}$ & $4,98 \pm 0,37^{\mathrm{b}}$ \\
IA (\%) & 76,66 & 73,33 & 73,55 & 66,66 & 55,33 \\
\hline
\end{tabular}




\begin{tabular}{|c|c|c|c|c|c|}
\hline $\begin{array}{l}\text { Sabor } \\
\text { residual }\end{array}$ & $6,52 \pm 0,18^{a}$ & $6,35 \pm 0,23^{a}$ & $6,45 \pm 0,30^{a}$ & $6,12 \pm 0,29^{a}$ & $4,70 \pm 0,32^{b}$ \\
\hline IA (\%) & 72,44 & 70,55 & 71,66 & 68,00 & 52,22 \\
\hline Cor & $7,33 \pm 0,21^{\mathrm{a}}$ & $7,23 \pm 0,21^{\mathrm{a}}$ & $7,47 \pm 0,20^{\mathrm{a}}$ & $7,00 \pm 0,23^{\mathrm{a}}$ & $7,21 \pm 0,21^{\mathrm{a}}$ \\
\hline IA (\%) & 81,44 & 80,33 & 83,00 & 77,77 & 80,11 \\
\hline $\begin{array}{c}\text { Aceitação } \\
\text { Global }\end{array}$ & $6,75 \pm 0,16^{a}$ & $6,62 \pm 0,24^{a}$ & $6,55 \pm 0,26^{a}$ & $6,25 \pm 0,29^{a}$ & $4,90 \pm 0,35^{\mathrm{b}}$ \\
\hline IA (\%) & 75,00 & 73,55 & 72,77 & 69,44 & 54,44 \\
\hline $\begin{array}{l}\text { Intenção de } \\
\text { Compra }\end{array}$ & $3,62 \pm 0,12^{\mathrm{a}}$ & $3,58 \pm 0,15^{a}$ & $3,55 \pm 0,16^{a}$ & $3,45 \pm 0,16^{a}$ & $2,58 \pm 0,18^{b}$ \\
\hline IA (\%) & 72,4 & 71,6 & 71,00 & 69,00 & 51,6 \\
\hline $\begin{array}{l}\text { Somatório } \\
\text { de notas* }\end{array}$ & $259^{a}$ & $239^{a}$ & $167^{\mathrm{b}}$ & $153^{\mathrm{b}}$ & $87^{c}$ \\
\hline \multicolumn{6}{|l|}{ Etapa 2} \\
\hline Aparência & $7,11 \pm 0,15^{\mathrm{a}}$ & $7,13 \pm 0,15^{a}$ & $7,09 \pm 0,16^{a}$ & $7,14 \pm 0,13^{\mathrm{a}}$ & $7,02 \pm 0,13^{a}$ \\
\hline IA (\%) & 79,00 & 79,22 & 78,77 & 79,33 & 78,00 \\
\hline Aroma & $6,76 \pm 0,19^{a}$ & $6,60 \pm 0,19^{a}$ & $6,27 \pm 0,22^{\mathrm{a}}$ & $6,61 \pm 0,20^{\mathrm{a}}$ & $6,28 \pm 0,18^{a}$ \\
\hline IA (\%) & 75,11 & 73,33 & 69,66 & 73,44 & 69,77 \\
\hline Sabor & $6,69 \pm 0,22^{\mathrm{a}}$ & $6,44 \pm 0,23^{\mathrm{ab}}$ & $6,42 \pm 0,29^{\mathrm{ab}}$ & $6,30 \pm 0,26^{\mathrm{ab}}$ & $5,70 \pm 0,28^{b}$ \\
\hline IA (\%) & 74,33 & 71,55 & 71,33 & 70,00 & 63,33 \\
\hline $\begin{array}{l}\text { Sabor } \\
\text { residual }\end{array}$ & $6,54 \pm 0,21^{a}$ & $6,58 \pm 0,21^{a}$ & $6,36 \pm 0,27^{a}$ & $6,22 \pm 0,25^{a}$ & $5,23 \pm 0,31^{b}$ \\
\hline IA (\%) & 72,66 & 73,11 & 70,66 & 69,11 & 58,11 \\
\hline Cor & $7,35 \pm 0,17^{a}$ & $7,30 \pm 0,17^{\mathrm{a}}$ & $7,26 \pm 0,16^{a}$ & $7,25 \pm 0,18^{a}$ & $7,23 \pm 0,16^{a}$ \\
\hline IA (\%) & 81,66 & 81,11 & 80,66 & 80,55 & 80,33 \\
\hline $\begin{array}{c}\text { Aceitação } \\
\text { Global }\end{array}$ & $6,52 \pm 0,21^{\mathrm{a}}$ & $6,39 \pm 0,23^{a}$ & $6,32 \pm 0,26^{a}$ & $6,46 \pm 0,24^{a}$ & $5,27 \pm 0,27^{\mathrm{b}}$ \\
\hline IA (\%) & 72,44 & 71,00 & 70,22 & 71,77 & 58,55 \\
\hline $\begin{array}{c}\text { Intenção de } \\
\text { Compra }\end{array}$ & $3,67 \pm 0,12^{\mathrm{a}}$ & $3,69 \pm 0,14^{a}$ & $3,50 \pm 0,17^{\mathrm{a}}$ & $3,43 \pm 0,15^{a}$ & $2,76 \pm 0,16^{b}$ \\
\hline IA (\%) & 73,40 & 73,80 & 70,00 & 68,60 & 55,20 \\
\hline $\begin{array}{c}\text { Somatório } \\
\text { de notas* }\end{array}$ & $237^{a}$ & $214^{a}$ & $176^{\mathrm{b}}$ & $143^{\mathrm{bc}}$ & $140^{c}$ \\
\hline
\end{tabular}

Letras diferentes na linha indicam diferença significativa pelo teste de Tukey $(p<0,05)$; EPM: Erro Padrão da Média; Etapa 1: F1 (0,40\% de $\mathrm{NaCl}$ ), F2 (0,30\% de $\mathrm{NaCl}$ e 0,10\% de $\mathrm{KCl}$ ), $\mathrm{F3}(0,20 \%$ de $\mathrm{NaCl}$ e 0,20\% de $\mathrm{KCl}), \mathrm{F} 4(0,10 \%$ de $\mathrm{NaCl}$ e 0,30\% de $\mathrm{KCl}$ ) e F5 (0,40\% de KCl); Etapa 2: F1 (0,40\% de $\mathrm{NaCl}+0,30 \%$ de GM), F2 (0,30\% de $\mathrm{NaCl}$ e $0,10 \%$ de $\mathrm{KCl}+0,30 \%$ de GM), F3 (0,20\% de $\mathrm{NaCl}$ e $0,20 \%$ de $\mathrm{KCl}+0,30 \%$ de $\mathrm{GM}), \mathrm{F} 4(0,10 \%$ de $\mathrm{NaCl}$ e $0,30 \%$ de $\mathrm{KCl}+0,30 \%$ de $\mathrm{GM})$ e $\mathrm{F5}(0,40 \%$ de $\mathrm{KCl}+0,30 \%$ de $\mathrm{GM})$; Diferença mínima significativa (DMS) entre as amostras com valor $\geq 34$ apresentam diferença estatística $(p<0,05)$, segundo a Tabela de Christensen (DUTCOSKI, 2011) para 60 julgamentos e 5 amostras.

Não houve diferença significativa $(\mathrm{p}>0,05)$ entre as formulações da Etapa 1 para os atributos aparência, aroma e cor. Resultados semelhantes foram verificados por Gomes et al. (2011), avaliando queijos do tipo Minas Frescal, com substituição de $\mathrm{NaCl}$ por $\mathrm{KCl}$ (0 a 75\%). Possivelmente, esse efeito ocorreu devido ao $\mathrm{KCl}$ também ser um sal com propriedades muito similares ao $\mathrm{NaCl}$, além de ser considerado seguro e possuir uma eficiência antimicrobiana equivalente ao $\mathrm{NaCl}$ (BIDLAS e LAMBERT, 2008). 
Menores notas foram verificadas para F5 nos atributos sabor residual, aceitação global e intenção de compra, sem diferença entre as demais formulações (Etapa 1). Este resultado pode ser explicado em função da ausência de sódio nessa formulação, o que evidenciou o gosto amargo proveniente do potássio, proporcionando uma menor aceitabilidade. Além disso, a total exclusão de sódio no produto pode aumentar a proteólise, acidez e atividade de água, interferindo na firmeza de produtos como o requeijão (CRUZ et al., 2011). Dessa forma, misturas entre os sais são as mais indicadas, pois ocorre uma interação entre o sódio e o potássio com as células receptoras gustativas ou com os mecanismos de transdução de sabor. Neste processo, o sódio se destaca, mascarando o sabor metálico do potássio (GOMES et al., 2011).

No atributo sabor F1, F2 e F3 foram mais aceitas que F5, isso porque o $\mathrm{NaCl}$ é a única substância capaz de oferecer um sabor salgado puro. Uma de suas mais importantes funções é criar uma base de percepção, intensificando e inibindo sabores, além de promover maior percepção do aroma (CRUZ et al., 2011). Conforme explicam Liem et al. (2011), as células gustativas apresentam receptores específicos para o sódio, que são ativados com baixas concentrações deste mineral, proporcionando a percepção do sabor salgado. Existem, porém, outros canais que são responsáveis por outros minerais, o que desencadeia diferentes sabores, como, por exemplo, o sabor amargo formado pelo $\mathrm{KCl}$, o que explica as menores notas para os produtos contendo maiores teores de $\mathrm{KCl}$.

$\mathrm{Na}$ Etapa 2, de forma semelhante, não foram verificadas diferenças entre as formulações $(p>0,05)$ para os atributos aparência, aroma e cor. A amostra F5 recebeu menores notas $(p<0,05)$, em relação ao sabor residual, aceitação global e intenção de compra. Ressalta-se que no atributo sabor apenas a formulação controle apresentou nota mais elevada que F5, o que demonstra que a adição de GM elevou à aceitação do requeijão. Também, verifica-se que o uso do GM como ingrediente na Etapa 2 promoveu maiores notas absolutas em F5, comparadas a Etapa 1, principalmente nos atributos sabor, sabor residual, aceitação global e intenção de compra. Resultados similares foram observados por Grummer et al. (2013) em queijos com substituição de $60 \%$ de $\mathrm{NaCl}$ por $\mathrm{KCl}$ e em conjunto com GM (0,11\%). Este efeito ocorre porque o GM promove um aumento do sabor salgado, por ação do ácido glutâmico, que ocorre naturalmente em alimentos proteicos, por exemplo (RANGAN e BARCELOUX, 2009). Igualmente, Carvalho et al. (2011) afirmam que o GM confere ao alimento um sabor diferenciado - umami - que é percebido quando o realçador entra em contato com os receptores gustativos, proporcionando ao mesmo tempo redução do gosto amargo provocado pelo $\mathrm{KCl}$.

Os atributos aparência, aroma e cor alcançaram IAs $\geq 70 \%$ em todas as formulações (Etapa 1 e 2), classificando os produtos com boa aceitação sensorial (DUTCOSKY, 2011). Nos demais atributos, observa-se que, em geral, apenas as amostras F1, F2 e F3 alcançaram este índice. Contudo, as formulações de requeijão com teor reduzido de sódio, tornam-se viáveis, principalmente para crianças e adolescentes, que possuem uma elevada ingestão alimentar de sódio. Assim, o requeijão restrito em sódio poderá favorecer bons hábitos alimentares e colaborar com as novas medidas públicas, que visam um menor consumo desse mineral pela população brasileira (NILSON et al., 2012).

O teste de ordenação (Etapa 1) apontou F1 e F2 como as amostras mais salgadas ( $\mathrm{p}<0,05)$.Já F3 e F4, se mostraram mais salgadas que F5. Estes resultados sugerem que indivíduos habituados com uma dieta com elevados teores de sódio podem apresentar maior sensibilidade à produtos com teor reduzido deste mineral (DRAKE et al., 2011). Isso, porque $70 \%$ dos adolescentes 
brasileiros apresentam uma ingestão de sódio superior ao valor máximo de ingestão tolerável (UL - $2.300 \mathrm{mg} / \mathrm{dia}$ ) (IBGE, 2011).

$\mathrm{Na}$ Etapa 2, de forma semelhante, F1 e F2 foram indicadas como mais salgadas ( $\mathrm{p}<0,05)$, no entanto F5 foi semelhante a F4, fato que demonstra uma melhora das notas para este atributo com adição de GM. Segundo Reyes e Guillermo (2011), é necessário a adição de duas partes de GM para uma de $\mathrm{NaCl}$, para que o $\mathrm{GM}$ exerça sua função, mantendo o sabor salgado do alimento mesmo com a redução de sódio. Sendo assim, F5 recebeu menores notas mesmo após a adição do realçador.

Na Fig. 1 está representada a distribuição dos provadores pelos valores hedônicos obtidos na avaliação dos atributos aparência, aroma, sabor, sabor residual, cor e aceitação global das formulações nas Etapas 1 e 2.

Figura 1. Distribuição dos provadores pelos valores hedônicos obtidos na avaliação sensorial das formulações de requeijão com teor reduzido de cloreto de sódio (NaCl) - Etapa 1 e Etapa 2.

\section{Etapa 1}
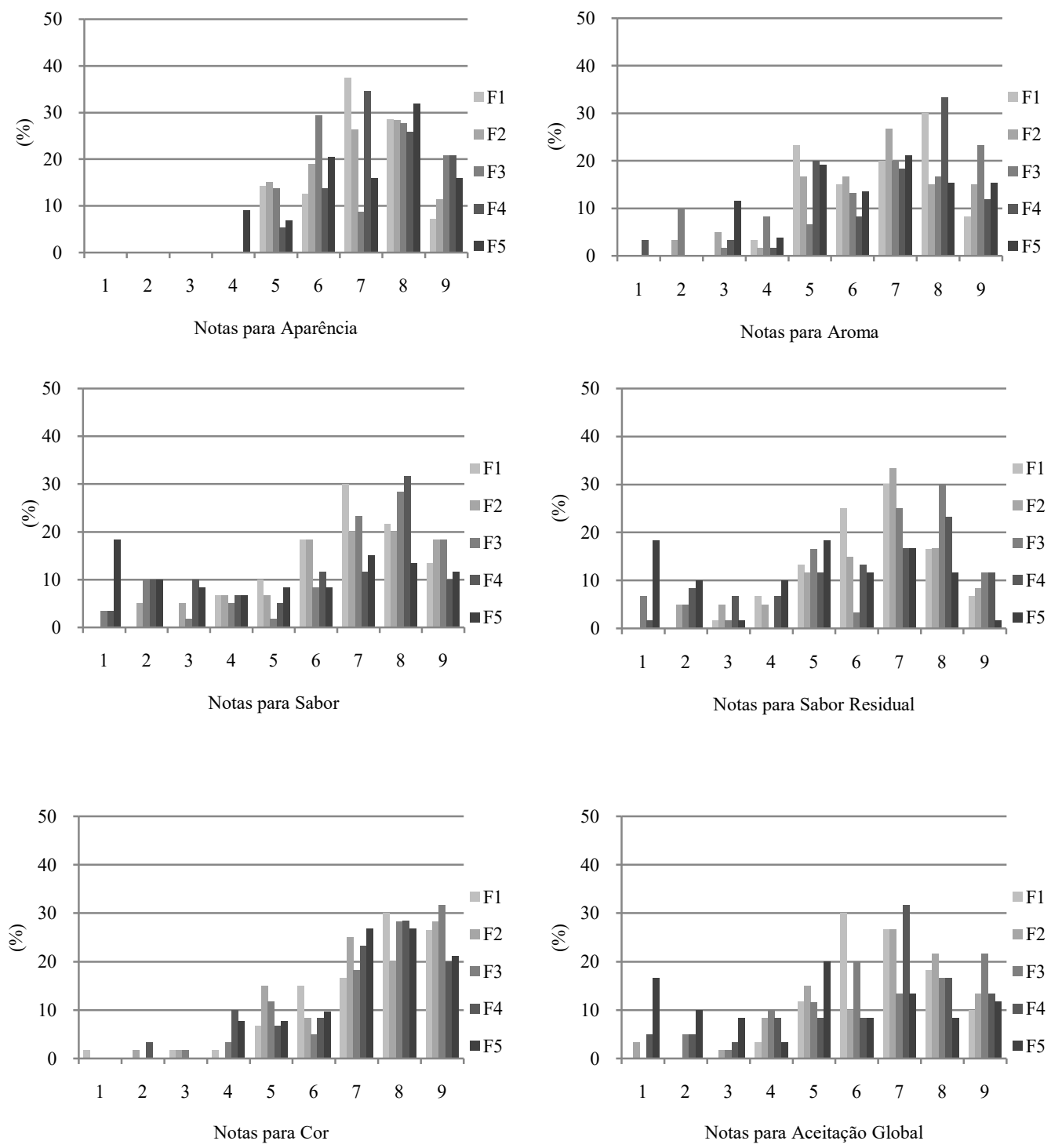


\section{Etapa 2}
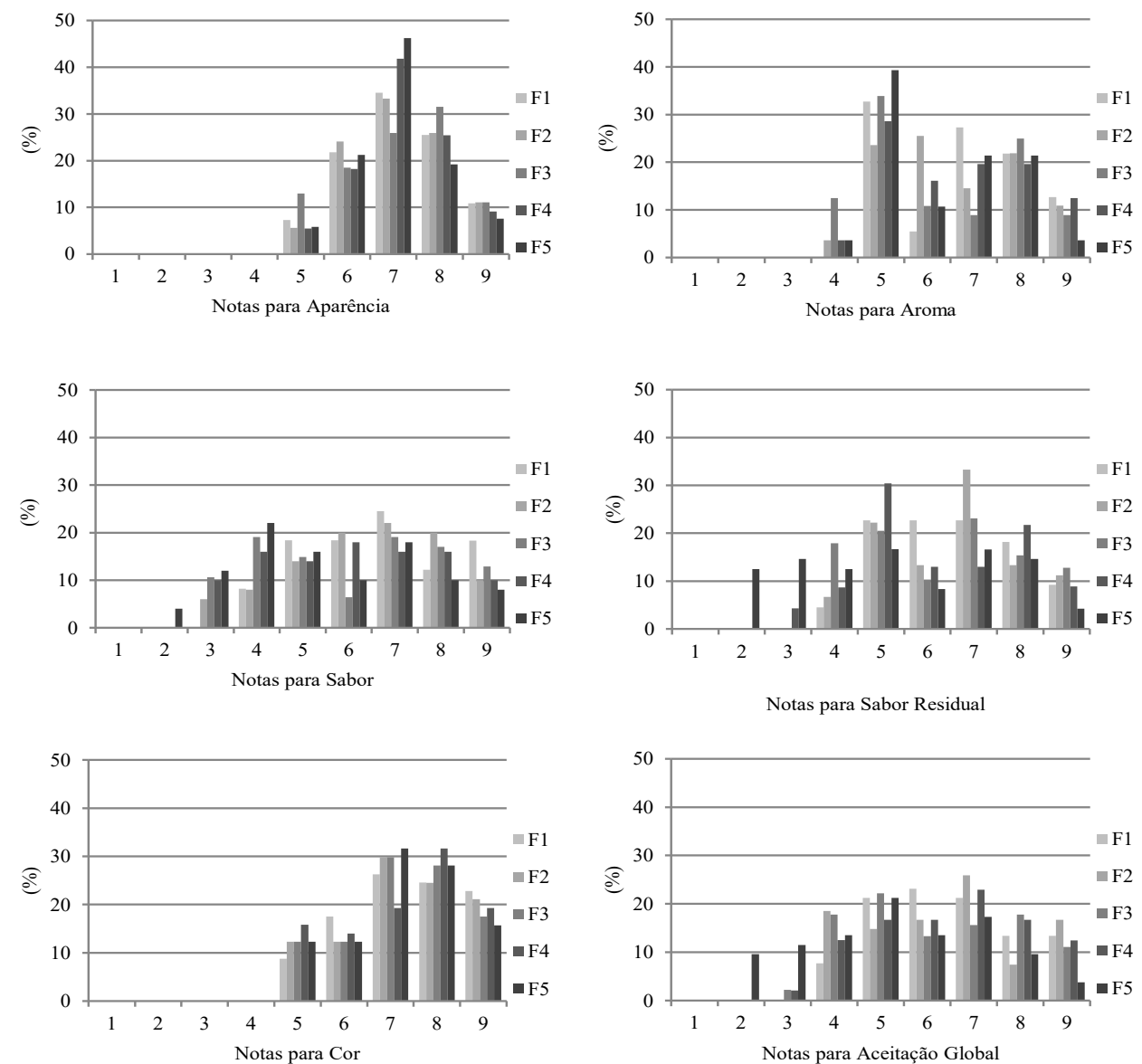

Legenda: Etapa 1: F1 (0,40\% de $\mathrm{NaCl}-100 \%), \mathrm{F} 2$ (0,30\% de $\mathrm{NaCl}-75 \%$ e 0,10\% de $\mathrm{KCl}-25 \%), \mathrm{F3}(0,20 \%$ de $\mathrm{NaCl}-50 \%$ e $0,20 \%$ de $\mathrm{KCl}-50 \%), \mathrm{F} 4$ (0,10\% de $\mathrm{NaCl}-25 \%$ e 0,30\% de $\mathrm{KCl}-75 \%)$ e $\mathrm{F} 5$ (0,40\% de $\mathrm{KCl}-100 \%)$; Etapa 2: $\mathrm{F} 1(0,40 \%$ de $\mathrm{NaCl}+0,30 \%$ de GM), F2 (0,30\% de $\mathrm{NaCl}$ e $0,10 \%$ de $\mathrm{KCl}+0,30 \%$ de GM), $\mathrm{F3}(0,20 \%$ de $\mathrm{NaCl}$ e $0,20 \%$ de $\mathrm{KCl}+0,30 \%$ de GM), F4 (0,10\% de $\mathrm{NaCl}$ e 0,30\% de $\mathrm{KCl}+0,30 \%$ de $\mathrm{GM}$ ) e $\mathrm{F} 5$ (0,40\% de $\mathrm{KCl}$ $+0,30 \%$ de GM).

Em geral, a maioria dos provadores atribuiu notas acima de 7 ("gostei moderadamente") na Etapa 1. Entretanto, salienta-se a elevada porcentagem de notas $\leq$ que 5 ("nem gostei/ nem desgostei”) no atributo sabor (51,6\%), sabor residual (58,3\%) e aceitação global (58,3\%) para F5, evidenciando menor aceitação deste produto. Diante destes resultados, o nível de substituição de $\mathrm{NaCl}$ por $\mathrm{KCl}$ deve ser restrito em virtude do gosto amargo produzido nos produtos, isso quando não são utilizados outros ingredientes melhoradores de sabor. Segundo El-Bakry e Sheehan (2014), em queijos com sabor suave, como o branco, obtém-se um bom resultado sensorial com a adição de até $50 \%$ de $\mathrm{KCl}$, sendo que o sabor amargo é mascarado pela quantidade de $\mathrm{NaCl}$ restante. Este fato pode explicar a atribuição de notas mais elevadas às formulações F2, F3 e F4.

Em relação à Etapa 2, a adição do GM elevou a frequência de notas $\geq$ a 5 em todos os atributos, demonstrando maior aceitação dos requeijões contendo $\mathrm{KCl}$. Assim, o GM pode ser adicionado ao produto como um sal de reforço, visto que aumenta a palatabilidade. Além disso, proporciona um gosto característico umami quando sofre hidrólise durante o processamento (JINAP e HAJEB, 2010).

\section{Composição físico-química}

Foram verificados os seguintes valores médios ( \pm desvio padrão) para a análise de umidade (\%) e

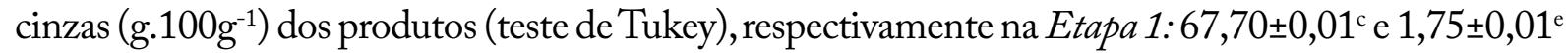
(F1); 70,26 $\pm 0,01^{\mathrm{a}}$ e $2,15 \pm 0,02^{\text {bc }}(\mathrm{F} 2) ; 69,00 \pm 0,01^{\mathrm{b}}$ e $1,97 \pm 0,01^{\mathrm{d}}(\mathrm{F} 3) ; 67,76 \pm 0,05^{\mathrm{c}}$ e $1,92 \pm 0,06^{\mathrm{d}}(\mathrm{F} 4)$; 
$68,93 \pm 0,04^{\mathrm{b}}$ e $1,95 \pm 0,01^{\mathrm{d}}(\mathrm{F} 5)$. Na Etapa 2 foram verificados os seguintes resultados, respectivamente: $65,34 \pm 0,07^{\text {de }}$ e $2,32 \pm 0,02^{\text {a }}(\mathrm{F} 1) ; 65,53 \pm 0,05^{\mathrm{d}}$ e $2,23 \pm 0,01^{\text {ab }}(\mathrm{F} 2) ; 67,85 \pm 0,07^{\mathrm{c}}$ e $2,17 \pm 0,06^{\text {bc }}(\mathrm{F} 3)$; $65,23 \pm 0,02^{\mathrm{e}}$ e $2,29 \pm 0,03^{\mathrm{a}}(\mathrm{F} 4) ; 64,87 \pm 0,04^{\mathrm{f}}$ e $2,13 \pm 0,02^{\mathrm{c}}(\mathrm{F} 5)$. Maiores teores de umidade (Etapa 1$)$ foram verificados para F2, seguidos por F3 e F5, contudo F1 e F4 tiveram os menores resultados. Todas as formulações apresentaram teores de umidade superiores ao recomendado pela Portaria $n^{\circ}$ 359, de 04 de setembro de 1997 (BRASIL, 1997), que aprova o regulamento técnico para fixação de identidade e qualidade de requeijão, que é de $65 \%$. Ressalta-se que valores de umidade acima de $55 \%$ em queijo minas frescal, podem torna o produto altamente perecível, o que pode ocorrer também no caso de requeijões (SALOTTI et al., 2006). Este fato reduz o shelf-life dos produtos, devendo ser consumido nos primeiros quinze dias após sua fabricação, mesmo sob refrigeração adequada, visto que são submetidos a um processamento mínimo.

$\mathrm{Na}$ Etapa 2 menores teores de umidade foram encontrados em F5 e maiores em F3, seguidos por F1 e F2 (p<0,05). Segundo Borin et al. (2008), o NaCl apresenta elevado potencial de retenção de água. $\mathrm{O}$ inverso ocorre com o GM, que eleva a perda de água nos produtos alimentícios, visto que, apesar de sua alta solubilidade em água e estabilidade a altas temperaturas e a luz, possui baixa higroscopicidade (JINAP e HAJEB, 2010), efeitos que podem explicar a baixa umidade das formulações adicionadas de GM.

Quanto aos teores de cinzas (Etapa 1), o maior conteúdo foi encontrado em F2, seguido por F3, F4 e F5, que não apresentaram diferença significativa entre si, enquanto que o menor teor foi verificado em $\mathrm{F} 1$. Essa variação está relacionada às diferentes concentrações de $\mathrm{NaCl}$ e $\mathrm{KCl}$ adicionadas a massa durante o processo de fabricação do requeijão (BITTENCOURT et al., 2013). Dados que corroboram com Broch et al. (2014), avaliando queijo mussarela com $100 \%$ de $\mathrm{NaCl}$ que apresentou menor teor de cinzas. Este fato pode ser explicado, pois os produtos que contêm uma maior combinação dos sais apresentam maior concentração de minerais em sua composição química.

$\mathrm{Na}$ Etapa 2, a amostra F5 apresentou a menor quantidade $(\mathrm{p}<0,05)$ de cinzas, sendo o maior teor nas demais formulações ( $p>0,05)$. Destaca-se que, em geral, a adição de GM aos requeijões elevou o conteúdo de cinzas. Resultados similares foram encontrados por Grummer et al. (2013). Este efeito pode ser explicado, pois o GM contém 13\% de sódio em sua composição química (CARVALHO et al., 2011), fato que elevou a concentração de minerais nos produtos.

$\mathrm{Na}$ Tabela 2 está descrita a composição físico-química e valores diários recomendados (VD) do requeijão padrão, comparado com um produto referência.

\section{Tabela 2. Composição físico-química e valores diários recomendados - VD* (porção média de $30 \mathrm{~g}$ ) do requeijão padrão (F1) sem glutamato monossódico, comparados com um produto comercial de referência**}

\begin{tabular}{lccc}
\hline \multicolumn{1}{c}{ Avaliação } & Média \pm DP & VD (\%)* & Referência $^{* *}$ \\
\hline Proteínas $\left(\mathrm{g} \cdot 100 \mathrm{~g}^{-1}\right)^{* * * *}$ & $8,90 \pm 0,07$ & 3,45 & 9,60 \\
Lipídios $\left(\mathrm{g} \cdot 100 \mathrm{~g}^{-1}\right)^{* * * *}$ & $19,40 \pm 0,09$ & 7,18 & 23,40 \\
Carboidratos $\left(\mathrm{g} \cdot 100 \mathrm{~g}^{-1}\right)^{* * * *}$ & $2,30 \pm 0,15$ & 0,23 & 2,40 \\
Calorias $\left(\mathrm{kcal} \cdot 100 \mathrm{~g}^{-1}\right)^{* * *}$ & $220,03 \pm 0,58$ & 2,98 & 257,00 \\
Fibra alimentar $\left(\mathrm{g} \cdot 100 \mathrm{~g}^{-1}\right)^{* * *}$ & 0,00 & - & 0,00 \\
\hline
\end{tabular}

Letras diferentes na linha indicam diferença significativa pelo teste de $t$ de student $(p<0,05) ;{ }^{*} V D$ : nutrientes avaliados pela média da DRI (2005), com base numa dieta de 2.214,00 kcall dia; **Valores comparados com requeijão cremoso (TACO, 2011); **Valores calculados em base úmida; DP: desvio padrão da média. 
Resultados superiores aos do presente estudo foram relatados por Grummer et al. (2013) em relação as proteínas e os lipídios. Fato similar foi observado para todas as avaliações quando se comparou F1 ao produto de referência (TACO, 2011), o que pode ser explicado pelos diferentes ingredientes utilizados nas formulações. Destaca-se que o teor de lipídios do requeijão formulado nesta pesquisa está abaixo do recomendado pela Legislação Brasileira (55\%) (BRASIL, 1997). Contudo, conforme a RDC no. 54 de 12 de novembro de 2012 (BRASIL,2012), pode-se classificar o requeijão desta pesquisa como light, já que houve redução de mais de $25 \%$ do seu teor lipídico comparado ao produto comercializado.

$\mathrm{Na}$ Tabela 3 estão descritos os teores médios de sódio e potássio das cinco formulações de requeijão avaliadas.

\section{Tabela 3. Composição físico-química (sódio - Na e potássio - K) e valores diários recomendados - VD (porção média de $30 \mathrm{~g}$ ) das formulações de requeijão, Etapas 1 e 2}

\begin{tabular}{|c|c|c|c|c|c|c|c|c|c|c|}
\hline & $\begin{array}{c}\text { F1 } \\
(\mathrm{mg} / 100 \mathrm{~g})\end{array}$ & $\begin{array}{l}\text { VD } \\
(\%)\end{array}$ & $\begin{array}{c}\mathrm{F} 2 \\
(\mathrm{mg} / 100 \mathrm{~g})\end{array}$ & $\begin{array}{l}\text { VD } \\
(\%)\end{array}$ & $\begin{array}{c}\text { F3 } \\
(\mathrm{mg} / 100 \mathrm{~g})\end{array}$ & $\begin{array}{l}\text { VD } \\
(\%)\end{array}$ & $\begin{array}{c}\mathrm{F} 4 \\
(\mathrm{mg} / 100 \mathrm{~g})\end{array}$ & $\begin{array}{l}\text { VD } \\
(\%)\end{array}$ & $\begin{array}{c}\text { F5 } \\
(\mathrm{mg} / 100 \mathrm{~g})\end{array}$ & $\begin{array}{l}\text { VD } \\
\text { (\%) }\end{array}$ \\
\hline & Média \pm DP & & Média \pm DP & & Média $\pm D P$ & & Média $\pm \mathrm{DP}$ & & Média $\pm D P$ & \\
\hline \multicolumn{11}{|c|}{ Etapa 1} \\
\hline $\mathrm{Na}$ & $220,84 \pm 0,05^{a}$ & 3,31 & $180,84 \pm 0,08^{b}$ & 2,71 & $140,84 \pm 0,07^{\mathrm{c}}$ & 2,11 & $\begin{array}{c}100,84 \pm \\
0,10^{\mathrm{d}}\end{array}$ & 1,51 & $60,84 \pm 0,04^{e}$ & 0,91 \\
\hline $\begin{array}{c}\% \\
\text { redução }\end{array}$ & 0,00 & & 18,11 & & 36,23 & & 54,34 & & 72,45 & \\
\hline K & $70,75 \pm 0,10^{\mathrm{e}}$ & 0,86 & $211,72 \pm 0,09^{d}$ & 2,59 & $236,90 \pm 0,08^{c}$ & 2,89 & $\begin{array}{c}261,59 \pm \\
0,09^{\mathrm{b}}\end{array}$ & 3,20 & $283,80 \pm 0,07^{a}$ & 3,47 \\
\hline $\begin{array}{c}\% \\
\text { aumento }\end{array}$ & 0,00 & & 199,25 & & 239,08 & & 269,74 & & 301,13 & \\
\hline \multicolumn{11}{|c|}{ Etapa 2} \\
\hline $\mathrm{Na}$ & $257,74 \pm 0,10^{a}$ & 3,15 & $214,74 \pm 0,04^{\mathrm{b}}$ & 2,62 & $177,74 \pm 0,06^{c}$ & 2,17 & $\begin{array}{c}137,74 \pm \\
0,04^{\mathrm{d}}\end{array}$ & 1,68 & $97,74 \pm 0,08^{\mathrm{e}}$ & 1,19 \\
\hline $\begin{array}{c}\% \\
\text { aumento/ } \\
\text { redução }\end{array}$ & $16,71^{\mu}$ & & $2,76^{\mu}$ & & $19,52^{\mu}$ & & $37,63^{\mu}$ & & $55,74^{\mu}$ & \\
\hline
\end{tabular}

Resultados em base úmida; Letras diferentes na linha indicam diferença significativa pelo teste de Tukey $(p<0,05)$; DP: Desvio Padrão da Média; VD: nutrientes avaliados considerando-se uma dieta diária de $2.000 \mathrm{mg}$ de $\mathrm{Na}$ (WHO, $2011)^{\text {e }} 2.455 \mathrm{mg}$ de $\mathrm{K}$ (DRI, 2005); ${ }^{\mu} \%$ de aumento em relação à $F 1$ (Etapa 1); ${ }^{\beta} \%$ de redução em relação à $F 1$ (Etapa 1); Etapa 1: F1 (0,40\% de $\mathrm{NaCl}), \mathrm{F} 2$ (0,30\% de $\mathrm{NaCl}$ e 0,10\% de $\mathrm{KCl}$ ), $\mathrm{F3}(0,20 \%$ de $\mathrm{NaCl}$ e $0,20 \%$ de $\mathrm{KCl}$ ), F4 (0,10\% de $\mathrm{NaCl}$ e 0,30\% de $\mathrm{KCl}$ ) e F5 (0,40\% de $\mathrm{KCl})$; Etapa 2: F1 (0,40\% de $\mathrm{NaCl}+0,30 \%$ de GM), F2 (0,30\% de $\mathrm{NaCl}$ e 0,10\% de $\mathrm{KCl}+0,30 \%$ de GM), $\mathrm{F3}(0,20 \%$ de $\mathrm{NaCl}$ e 0,20\% de $\mathrm{KCl}+0,30 \%$ de GM), $\mathrm{F} 4(0,10 \% \mathrm{de} \mathrm{NaCl}$ e $0,30 \%$ de $\mathrm{KCl}+0,30 \%$ de $\mathrm{GM})$ e $F 5(0,40 \%$ de $\mathrm{KCl}+0,30 \%$ de $\mathrm{GM})$.

Sabendo-se que a formulação F4 (Etapa 1 e 2) obteve melhor aceitabilidade sensorial (Tabela 1), isto é maior teor de adição de $\mathrm{KCl}$ e aceitação semelhante ao padrão, foi possível uma redução média de 54\% de sódio em relação ao padrão. Resultados similares foram observados por Gomes et al. (2011), sendo possível uma redução de 51,8\% de sódio sem interferir no sabor dos produtos.

Em virtude da importância do cloreto de sódio para a indústria, seja na conservação, melhora do sabor e outros efeitos congruentes dos produtos, a redução desse sal torna-se um desafio, visto que a ingestão excessiva desse mineral está associada ao aumento da pressão arterial, principal causadora de doenças cardiovasculares, assim como câncer gástrico, redução da densidade mineral óssea e obesidade (LIEM et al., 2011). 


\section{Conclusão}

Foi possível reduzir o teor de $\mathrm{NaCl}$ e elevar o conteúdo de $\mathrm{KCl}$ nas formulações de requeijão sem interferir na aceitação sensorial com um nível de adição de até 0,30\% de $\mathrm{KCl}$ (redução de $75 \%$ de $\mathrm{NaCl}$ ), sendo que a adição de $\mathrm{GM}$ elevou, em geral, a aceitação dos produtos. Assim sendo, é possível restringir o teor de $\mathrm{NaCl}$ em requeijão, principalmente através de sua utilização em conjunto com o $\mathrm{KCl}$ e GM, o que, futuramente, poderá reduzir o risco do desenvolvimento de patologias nos indivíduos.

\section{Agradecimentos}

Os autores agradecem ao Programa Institucional de Atividades Extensionistas (PIAE) da UNICENTRO pela concessão da bolsa.

\section{Referências}

AGARWAL, S.D.; MCCOY,D.; GRAVES, W.; GERARD, P.D.; CLARCK, S. Sodium content in retail Cheddar, Mozzarella, and process cheeses varies considerably in the United States. Journal of Diary Science, Champaign, v.94, n.3, p.1605-1615, 2011.

ALINO, M.; GRAU, R.; TOLDRÁ, F.; BLESA, E.; PAGÁN, M.J.; BARAT, J.M. Influence of sodium replacement on physicochemical properties of dry-cured loin. Meat Science, Barking, v.83, n.3, p.423-430, 2009.

ANDRADE, T.F. Importância das análises físico-químicas no controle de qualidade de alimentos consumidos em Santa Catarina. 2012. 32f. Monografia (Especialista em Saúde Pública) - Universidade Federal de Santa Catarina, Florianópolis, 2012.

AOAC International. Official Methods of Analysis of AOAC International. 18 ed. 4 rev. Gaithersburg (MD): AOAC, 2011.1505p.

BIDLAS, E.; LAMBERT, R.J.W. Comparing the antimicrobial effectiveness of $\mathrm{NaCl}$ and $\mathrm{KCl}$ with a view to salt/sodium replacement. Internacional Journal of Food Microbiology, Amsterdam , v.124, n.1, p.98-102, 2008.

BITTENCOURT, R.H.F.P.M.; CORTEZ,M.A.S.; MÁRSICO,E.T.; ROSA, R.M.S.S.; TAXI, C.M.A.D.; FATURI, C. Caracterização de Requeijão Marajoara e Minas Frescal produzidos com leite de búfalas no Estado do Pará, Brasil. Ciência Rural, Santa Maria, v.43, n.9, p.1687-1692, 2013.

BLIGH, E.G.; DYER, W.J. A rapid method of total lipid extraction and purification. Canadian Journal Biochemistry and Physiology, Ottawa, v.37, n.8, p.911-917, 1959. 
BORIN, I.; FRASCARELLI, E.C.; MAURO, M.A.; KIMURA, M. Efeito do prétratamento osmótico com sacarose e cloreto de sódio sobre a secagem convectiva de abóbora. Ciência e Tecnologia de Alimentos, Campinas, v.28, n.1, p.39-50, 2008.

BRASIL. Agência Nacional de Vigilância Sanitária (ANVISA). Resolução RDC no 54, de 12 de novembro de 2012. Dispõe sobre o Regulamento Técnico sobre Informação Nutricional Complementar. Diário Oficial da União. Poder Executivo, Brasília, DF, nov. 2012.

BRASIL. Ministério da Agricultura e do Abastecimento. Portaria no 359, de 04 de setembro de 1997. Regulamento Técnico para Fixação de Identidade e Qualidade do Requeijão ou Requesõn. Diário Oficial da União. Poder Executivo, Brasília, DF, set.1997.

BROCH, A.N.; CARVALHO, C.B.; MADRONA, G.S. Análise sensorial de queijo mussarela com reduzido teor de sódio. Revista GEINTEC, São Cristóvão, v.4, n.2, p.841-849, 2014.

CAMPANA, E.M.G.; BRANDÃO, A.A.; MAGALHÃES, ME.; FREITAS, E.V.de.; POZZAN, R.; BRANDÃO, A.P. Pré-hipertensão em crianças e adolescentes. Revista Brasileira de Hipertensão, Rio de Janeiro, v.16, n.2, p.92-102, 2009.

CARVALHO, P.R.R.M.; BOLOGNESI, V.J.; BARREIRA, S.M.W.; GARCIA, C.E.R. Características e segurança do glutamato monossódico como aditivo alimentar: artigo de revisão. Revista Visão Acadêmica, Curitiba, v.12, n.1, p.53-64, 2011.

COZZOLINO, S.M.F. Biodisponibilidade de Nutrientes. $4^{\mathrm{a}}$ ed. Barueri (SP): Manole, 2012.1334p.

CRUZ, A.G.; FARIA,J.A.F.; POLLONIO, M.A.R.; BOLINI,H.M.A.; CELEGHINI, R.M.S.; GRANATO, D. Review: Cheeses with reduced sodium content: Effects on functionality, public health benefits and sensory properties. Trends in Food Science \& Technology, Cambridge, v.22, n.6, p-276-291, 2011.

DIETARY REFERENCE INTAKES (DRI). Dietary reference intakes for energy, carbohydrate, fiber, fat, fatty acids, cholesterol, protein and amino acids. Washington (DC): The National Academies Press, 2005.1332p.

DRAKE, S.L.; LOPETCHARAT, K.; DRAKE, M.A. Salty taste in dairy foods: Can we reduce the salt?. Journal of Dairy Science, Champaign, v.94, v.2, p.636-645, 2011.

DUTCOSKY, S.D. Análise Sensorial de Alimentos. $3^{a}$ ed. Curitiba (PR): Champagnat, 2011.531p.

EL-BAKRY, M.; SHEEHAN, J. Analysing cheese microstructure: A review of recent developments. Journal of Food Engineering, London, v.125, n.3, p.84-96, 2014. 
GOMES, A.P.; CRUZ, A.G.; CADENA, R.S.; CELEGHINI, R.M.S.; FARIA, J.A.F.; BOLINI,H.M.A.; POLLONIO, M.A.R.; GRANATO, D. Manufacture of low-sodium minas fresh cheese: effect of the partial replacement of sodium chloride with potassium chloride. Journal of Dairy Science, Champaign, v.94, n.6, p.2701-270, 2011.

GRUMMER,J.; BOBOWSKI, N.; KARALUS, M.; VICKERS, Z.; SCHOENFUSS, T. Use of potassium chloride and flavor enhancers in low sodium Cheddars cheese. Journal of Dairy Science, Champaign, v.96, n.3, p.1401-1418, 2013.

INSTITUTO BRASILEIRO DE GEOGRAFIA E ESTATÍSTICA (IBGE). Pesquisa de Orçamentos Familiares 2008-2009: análise do consumo alimentar pessoal no Brasil. Rio de Janeiro (RJ): IBGE, 2011.150p.

JINAP, D.; HAJEB, P. Glutamate. Its applications in food and contribution to health. Appetite, London, v.55, n.1, p.1-10, 2010.

LIEM, D.G.; MIREMADI, F.; KEAST, R.S.J. Reducing Sodium in Foods: The Effect on Flavor. Nutrients, Basel, v.3, n.1, p.694-711, 2011.

MERRILL,A.L.; WATT, B.K. Energy value of foods: basis and derivation. Agricultural Handbook, Washington (DC): USDA, 1973. 106p.

MONTEIRO, C.A.; LEYY, R.B.; CLARO, R.M.; CASTRO, I.R.; CANNON, G. Increasing consumption of ultra-processed foods and likely impact on human health: evidence from Brazil. Public Health Nutrition, Wallingford, v.14, n.1, p.5-13, 2011.

NILSON, E.A.F.; JAIME, P.C.; RESENDE, D.O. Iniciativas desenvolvidas no Brasil para a redução do teor de sódio em alimentos processados. Revista Panamericana de Salud Pública, Washington, v.32, n.4, p.92-287, 2012.

ORGANIZAÇÃO MUNDIAL DE SAÚDE (OMS). Cardiovascular diseases (CVDs). 2015. Disponível em: <www.who.int/mediacentre/factsheets/fs317/en/index.html>. Acesso em: 05 dez. 2015.

POUSSEL, E.; MERMET, J. M. ICP Emission Spectrometers: Analytical Figures of Merit. Applied Spectroscopy, Plainfield, v.49, n.10, p.12A-18A, 1995.

PURDY,J.; ARMSTRONG, G.Dietary salt and the consumer: reported consumption and awareness of associated healthy risks. In: GUINEE, T.P.; O'KENNEDY, B.T. Reducing salt in foods: Practical strategies. Boca Raton (FL): CRC Press, 2007. p.99-123.

RANGAN, C.; BARCELOUX, D.G. Food additives and sensitivities. Disease a Month, Chicago, v.55, n.5, p.292-311, 2009. 
REYES, R.; GUILLERMO, F. Umami e glutamato: aspectos químicos, biológicos e tecnológicos. São Paulo (SP): Plêiade, 2011.195p.

RIBEIRO, V.F.; RIBEIRO, M.A.; VASCONCELOS, M.A.S.; ANDRADE, S.A.C.; STAMFORD, T.L.M. Processed foods aimed ar children and adolescents: Sodium content, adequacy according to the dietary reference intakes and label compliance. Revista de Nutrição, Campinas, v.26, n.4, p.397-406, 2013.

SALOTTI, B.M.; CARVALHO, A.C.F.B.; AMARAL, L.A.; VIDAL-MARTINS, A.M.C.; CORTEZ,A.L. Qualidade microbiológica do queijo minas frescal comercializado no município de Jaboticabal, SP, Brasil. Arquivos do Instituto Biológico, São Paulo, v.2, n.73, p.171-175, 2006.

TABELA BRASILEIRA DE COMPOSIÇÃO DOS ALIMENTOS (TACO). Tabela brasileira de composição dos alimentos. 4 ed. Revisada e ampliada. Campinas (SP): NEPA, 2011.164p.

TRAD, L.A.B.; TAVARES, J.S.C.; SOARES, C.S.; RIPARDO, R.C. Itinerários terapêuticos face à hipertensão arterial em famílias de classe popular. Cadernos de Saúde Pública, Rio de Janeiro, v.26, n.4, p.797-806, 2010.

VAN DENDER, A.G.F.; SPADOTI, L.M.; ZACARCHENCO, P.B.; TRENTO, F.K.H.S.; ORMENESE, R.C.S.C.; MORGANO, M.A. Efeito dos sais fundamentais nas características do requeijão cremoso sem adição de gordura e com teor reduzido de sódio. Revista do Instituto de Laticínios Cândido Tostes, Juiz de Fora, v.67, n.387, p.38-47, 2012.

WORLD HEALTH ORGANIZATION (WHO). Creating an enabling environment for population based salt reduction strategies: report of a Joint Technical Meeting held by WHO and FSA/UK. Geneva (SW): WHO, 2010. 44p.

WORLD HEALTH ORGANIZATION (WHO). Review and updating of current WHO recommendations on salt/sodium and potassium consumption. Geneva (SW): WHO, 2011. 8p. 